\title{
Aplicação em pré-colheita de cloreto de cálcio no controle do despencamento natural dos frutos de bananeira 'FHIA-18'
}

\author{
Calcium chloride in preharvest application to control the natural \\ fruit dropping of 'FHIA-18' banana tree
}

\begin{abstract}
Marlon Cristian Toledo Pereira ${ }^{I^{*}}$ Luiz Carlos Chamhum SalomãoII Rayane Carneiro dos Santos Sebastião de Oliveira e Silva ${ }^{\mathrm{II}}$ Paulo Roberto Cecon ${ }^{\mathrm{II}}$ Silvia Nietsche $^{\mathrm{I}}$
\end{abstract}

\section{RESUMO}

$O$ objetivo deste trabalho foi avaliar o efeito da aplicação de $\mathrm{CaCl}_{2}$ em pré-colheita, em diferentes doses e épocas no controle do despencamento natural de frutos da bananeira 'FHIA-18'. O delineamento experimental foi $o$ de blocos casualizados, em esquema fatorial $3 \times 3+1$ testemunha, sendo testadas as doses de 2, 4 e 6\% de $\mathrm{CaCl}$, aos 60, 90 e 120 dias após a emissão do cacho, com cinco repetições e uma planta por parcela. Observou-se redução linear da resistência ao despencamento e do tempo de maturação do fruto com o acréscimo das doses de $\mathrm{CaCl}_{2}$. Os frutos apresentaram maior firmeza com três aplicações de $\mathrm{CaCl}_{2}$. Os teores de cálcio da casca e da polpa não foram alterados significativamente com as doses e épocas de aplicações do $\mathrm{CaCl}_{2}$, indicando que o cálcio não é absorvido efetivamente pelo fruto. A aplicação de $\mathrm{CaCl}_{2}$ na pré-colheita não reduz o despencamento dos frutos da bananeira 'FHIA-18'.

Palavras-chave: Musa spp., $\mathrm{CaCl}_{2}$, queda de frutos, qualidade do fruto.

\section{ABSTRACT}

The objective of this research was to evaluate the effect of $\mathrm{CaCl}$, application in preharvest and in different doses and times in order to control the natural fruit dropping of banana 'FHIA-18'. The experimental design was in a randomized blocks, in a factorial scheme $3 \times 3+1$ control, with three doses of $\mathrm{CaCl}_{2}$ (2, 4 and 6\%), three times of application (60, 90 and 120 days) after the bunch emission, with five replicates and one plant per parcel. A linear decrease of dropping resistance and fruit time maturation with increasing doses of $\mathrm{CaCl}_{2}$ was observed. The fruits showed to be more consistent with three applications of $\mathrm{CaCl}_{2}$. The calcium content of the peel and pulp were not significantly changed with the doses and applications of $\mathrm{CaCl}_{2}$, indicating that calcium is not absorbed effectively by the fruits. The application of $\mathrm{CaCl}_{2}$ in preharvest do not reduce the natural fruit dropping in banana 'FHIA-18'.

Key words: Musa spp., $\mathrm{CaCl}_{2}$, finger drop, fruit quality.

\section{INTRODUÇÃO}

O Brasil destaca-se como um dos principais produtores mundiais de banana, entretanto sua produção está concentrada emalgumas variedades dogrupo Prataeas do tipo Cavendish. Apesar dessas variedades apresentarem alto potencial produtivo, apresentam suscetibilidade às principais doenças causadas por fungos, dentre as quais destacamos sigatoka-amarela (Mycosphaerella musicola), sigatoka-negra (Mycosphaerella fijienses) e mal-do-panamá (Fusarium oxysporum f sp. cubense), esta última, mais especificamente, as do grupo Prata (ALVES, 1999; SANTOS et al., 2013).

Diante desse preocupante cenário, novos materiais foram recomendados como 'Thap Maeo', 'Caipira' ('Yangambi'), 'Prata-Baby' ('Nam'), 'Fhia Maravilha' ('FHIA-01'), 'FHIA 18', 'FHIA-21', 'Prata-Zulu' ('Pisang Awak'), 'Prata-Graúda' ('SH3640') e 'Bucanera' (CORDEIRO \& MOREIRA, 2006). A bananeira 'FHIA-18', híbrido de 'Prata Anã' (AAAB), produzido pela Fundação Hondurenha de Investigação Agrícola (FHIA), apresenta excelente produtividade, semelhante às bananeiras do subgrupo Cavendish, e tem como principal característica a

IDepartamento de Ciências Agrárias, Universidade Estadual de Montes Claros (UNIMONTES), 39440-000, Janaúba, MG, Brasil. E-mail: marlon.pereira@unimontes.br. *Autor para correspondência.

IIDepartamento de Fitotecnia, Universidade Federal de Viçosa (UFV), Viçosa, MG, Brasil.

IIIEmbrapa Mandioca e Fruticultura, Cruz das Almas, BA, Brasil. 
resistência à sigatoka-negra (HOLDERNESS et al., 2000; DONATO et al.,2009; JESUS et al., 2009). Entretanto, a alta susceptibilidade à queda natural e perecibilidade dos frutos tem limitado sua expansão (PEREIRA et al., 2004; SANTOS et al., 2006; DONATO et al., 2009).

A queda natural dos frutos, conhecido como "Finger drop" ou "Weakneck" é uma desordem fisiológica que promove a queda dos frutos durante o processo de amadurecimento. Esta queda está associada ao enfraquecimento da região do pedicelo, fazendo com que os frutos se separem facilmente da penca (PAULL, 1996; SAENGPOOK et al., 2007). Este fenômeno se soma as outras perdas que ocorrem durante o período de pós-colheita, trazendo prejuízos de ordem econômica, pois a comercialização internacional e nacional de bananas é realizada basicamente por pencas ou pencas subdivididas (buquê) (ALVES, 1999).

A sensibilidade ao despencamento pode ser minimizada por meio de manejo fitotécnico adequado. Alguns estudos têm demonstrado que o uso do etanol, ácido giberélico e o cloreto de cálcio $\left(\mathrm{CaCl}_{2}\right)$ na pós-colheita da banana podem controlar a queda natural dos frutos e prolongar a vida de prateleira (ESGUERRA et al., 1993; AGRAVANTE et al., 2000; RAO \& CHUNDAWAT, 1991; IMSABAI et al., 2006; SALAZAR \& SERRANO, 2013). Os autores sugerem que os frutos tratados com $\mathrm{CaCl}_{2}$ apresentam incremento na firmeza, ficando mais resistentes à queda natural.

O objetivo do presente trabalho foi avaliar o efeito da aplicação de $\mathrm{CaCl}_{2}$ em pré-colheita, em diferentes concentrações e épocas, no controle do despencamento natural de frutos da bananeira 'FHIA18 ' após a colheita.

\section{MATERIAL E MÉTODOS}

O experimento foi realizado em lote de agricultor, no município de Nova Porteirinha, MG. A área onde foi instalado o experimento possui as coordenadas geográficas $15^{\circ} 45^{\prime} 05^{\prime}$ S e $43^{\circ} 15^{\prime} 46^{\prime \prime}$ $\mathrm{W}$, com altitude de aproximadamente $540 \mathrm{~m}$. A pluviosidade média da região é de aproximadamente $870 \mathrm{~mm}$, temperatura média anual de $24^{\circ} \mathrm{C}$, umidade relativa média de $65 \%$ e clima Aw (tropical com chuva de verão), segundo a classificação de Köpen. As características químicas da camada de $0-20 \mathrm{~cm}$ do solo areno-argiloso foram $\mathrm{pH}$ em água 6,$0 ; 7,0 \mathrm{mg} \mathrm{dm}^{-3} \mathrm{de}$ $\mathrm{P} ; 50 \mathrm{mg} \mathrm{dm}^{-3} \mathrm{de} \mathrm{K} ; 3,0 \mathrm{cmol}_{\mathrm{c}} \mathrm{dm}^{-3} \mathrm{de} \mathrm{Ca} ; 1,1 \mathrm{cmol}_{\mathrm{c}} \mathrm{dm}^{-3}$ de $\mathrm{Mg} ; 0,1 \mathrm{cmol}_{\mathrm{c}} \mathrm{dm}^{-3}$ de $\mathrm{Al} ; 1,5 \mathrm{cmol}_{\mathrm{c}} \mathrm{dm}^{-3} \mathrm{de} \mathrm{H}+\mathrm{Al}$; $4,4 \mathrm{cmol}_{\mathrm{c}} \mathrm{dm}^{-3}$ de CTC efetiva e 5,8 $\mathrm{cmol}_{\mathrm{c}} \mathrm{dm}^{-3}$ de CTC total. Os tratos culturais, adubação e irrigação das bananeiras foram efetuados segundo as recomendações de ALVES (1999). Foram selecionadas 50 bananeiras 'FHIA-18' na fase de emissão de cacho (logo após o aparecimento na roseta) da planta-filha, conduzidas em espaçamento $3 \times 2 \mathrm{~m}$.

O delineamento experimental foi o de blocos casualizados, em esquema fatorial $3 \times 3+1$ testemunha, sendo testadas as doses de 2 , 4 e $6 \%$ de $\mathrm{CaCl}_{2}$ aos 60, 90 e 120 dias após a emissão do cacho, com cinco repetições e uma planta por parcela. Na aplicação do cloreto de cálcio, utilizou-se pulverizador costal manual, aplicando-o por cerca de 30 segundos em cada cacho até o escorrimento.

Os cachos foram colhidos no estádio descrito por ALVES (1999), quando os frutos apresentavam quinas ou angulosidades da superfície reduzidas e a coloração da casca passava do verdeescuro intenso para o verde mais claro, com diâmetro do fruto central da $2^{\text {a }}$ penca variando entre $34 \mathrm{~mm}$ e $38 \mathrm{~mm}$. Em seguida, a segunda, terceira e quarta pencas foram transportadas para um galpão coberto, onde os frutos foram individualizados das pencas. Selecionaram-se 20 frutos de cada planta para análise do despencamento, os quais foram lavados, utilizando 10 destes para as demais avaliações. A avaliação do despencamento foi feita utilizando um despencador mecanizado, composto basicamente por chassi de madeira, tracionador de metal, motor e penetrômetro manual. Quando a banana não resiste mais a força aplicada, o pedicelo se rompe na região do "ombro", próximo à polpa do fruto, cessando a pressão no penetrômetro, que registra a força necessária à ruptura do pedicelo. Em seguida, pesouse individualmente o fruto e mediu-se comprimento do fruto, calibração radial (diâmetro entre quinas do fruto), calibração lateral (diâmetro do fruto), comprimento e diâmetro do pedicelo. A seguir, as caixas de papelão contendo os frutos foram colocadas em galpão com monitoramento da temperatura.

Ao atingirem o estádio 6 de maturação (totalmente amarelo), os frutos foram novamente pesados individualmente, tendo os valores de perda de matéria fresca expressos em percentagem do peso inicial. Em seguida, efetuou-se o teste de resistência ao despencamento, para quantificar o grau de suscetibilidade ao despencamento natural de frutos, e mediu-se a firmeza dos frutos com casca. A casca e a polpa foram separadas e pesadas. Finalizando essa etapa, mediu-se a espessura da casca. Amostras do pedicelo e da região mediana da casca e da polpa do fruto foram submetidas à determinação da porcentagem de matéria seca e dos níveis de cálcio, potássio e magnésio. 
Para análise dos níveis de cálcio, potássio e magnésio, pesou-se $0,5 \mathrm{~g}$ de cada amostra, transferindoas em seguida para tubos de digestão, onde foram adicionados $6 \mathrm{~mL}$ de solução nitroperclórica $\left(\mathrm{HNO}_{3} \mathrm{e}\right.$ $\mathrm{HClO}_{4}$ na proporção de 2:1). Os tubos foram levados ao bloco digestor, aumentando a temperatura gradativamente até atingir $160^{\circ} \mathrm{C}$, os quais permaneceram por 40 minutos nessa temperatura. Aumentou-se a temperatura para $250^{\circ} \mathrm{C}$, deixando cerca de 20 minutos. Após esfriados, os extratos foram transferidos para balão volumétrico de $50 \mathrm{~mL}$, completados com água deionizada. Para as determinações, utilizou-se espectrofotômetro de absorção atômica. Efetuaram-se as leituras das soluções-padrão ou do extrato nítrico-perclórico, transferindo $0,5 \mathrm{~mL}$ desse extrato para copo plástico, acrescentando $22 \mathrm{~mL}$ de água deionizada e $2,5 \mathrm{~mL}$ da solução de óxido de lantânio $5 \%$ e homogeneizando. Os resultados foram expressos em gramas de cálcio por quilograma do tecido.

As características avaliadas foram submetidas à análise de variância, com desdobramentos das interações significativas, tendo os efeitos das concentrações testados e ajustados em equações de regressão, sendo os efeitos da época de aplicação comparados pelo teste de Tukey a $5 \%$ de probabilidade. As análises estatísticas foram efetuadas com o auxílio do Sistema de Análises Estatísticas e Genéticas da Universidade Federal de Viçosa (SAEG-UFV).

\section{RESULTADOS E DISCUSSÃO}

De acordo com a análise de variância, observou-se que não houve interações significativas entre concentração de $\mathrm{CaCl}_{2}$ e época de aplicação, a $5 \%$ de probabilidade, pelo teste $\mathrm{F}$, com relação à maioria das características avaliadas. Houve interação significativa apenas das características teor de potássio na casca e teor de cálcio e magnésio no pedicelo, as quais foram desdobradas.

Observou-se redução linear da resistência ao despencamento dos frutos com o incremento das doses de cloreto de cálcio, em aplicações no cacho em pré-colheita (Figura 1A). Esse comportamento pode ser explicado pela falta da efetiva infiltração proporcional do cálcio nos frutos em função das doses de cloreto de cálcio aplicadas, já que a expectativa seria de que ocorresse o inverso, ou seja, que maiores concentrações de cálcio proporcionassem maior resistência ao despencamento. Possivelmente, isso ocorreu também devido ao amadurecimento mais precoce dos frutos tratados com maiores doses de cloreto de cálcio e, portanto, esses frutos estariam mais sensíveis à queda (Figura 1B). No caso da bananeira 'FHIA 18', destacamos que dois componentes associados ao genoma da planta contribuem significativamente para a ocorrência da queda natural dos frutos. Um deles é a presença do genoma A em três dos quatros conjuntos cromossômicos (AAAB) e o outro refere-se ao fato de ser um tetraploide e, portanto, apresentar maior grau de suscetibilidade ao despencamento. Segundo SILVA (1999), trabalhando com imersão da banana 'Pioneira' em soluções contendo cálcio, o cálcio que penetrou nos frutos não foi capaz de alterar a resistência dos frutos ao despencamento, não justificando o uso desse elemento em imersão pós-colheita. Entretanto, estudo recente demonstrou que a associação do ácido giberélico (200ppm), com $\mathrm{CaCl}_{2}$ (4\%) ou etanol (60\% $\mathrm{v} / \mathrm{v}$ ) aplicados em pós-colheita na porção final do pedicelo de bananas 'Cuarenta Dias' foram efetivos no controle do despencamento. De acordo com os autores, não foi observado despencamento dos frutos até os 15 dias de armazenamento em temperatura ambiente (SALAZAR \& SERRANO, 2013).

O cálcio recebe considerável atenção devido ao seu efeito na redução da senescência dos frutos. Estudos indicam que, no amadurecimento dos frutos, a taxa de senescência frequentemente depende dos níveis de cálcio do tecido (AGHDAM et al., 2012). Ao contrário do que era esperado, observou-se redução linear no tempo de maturação

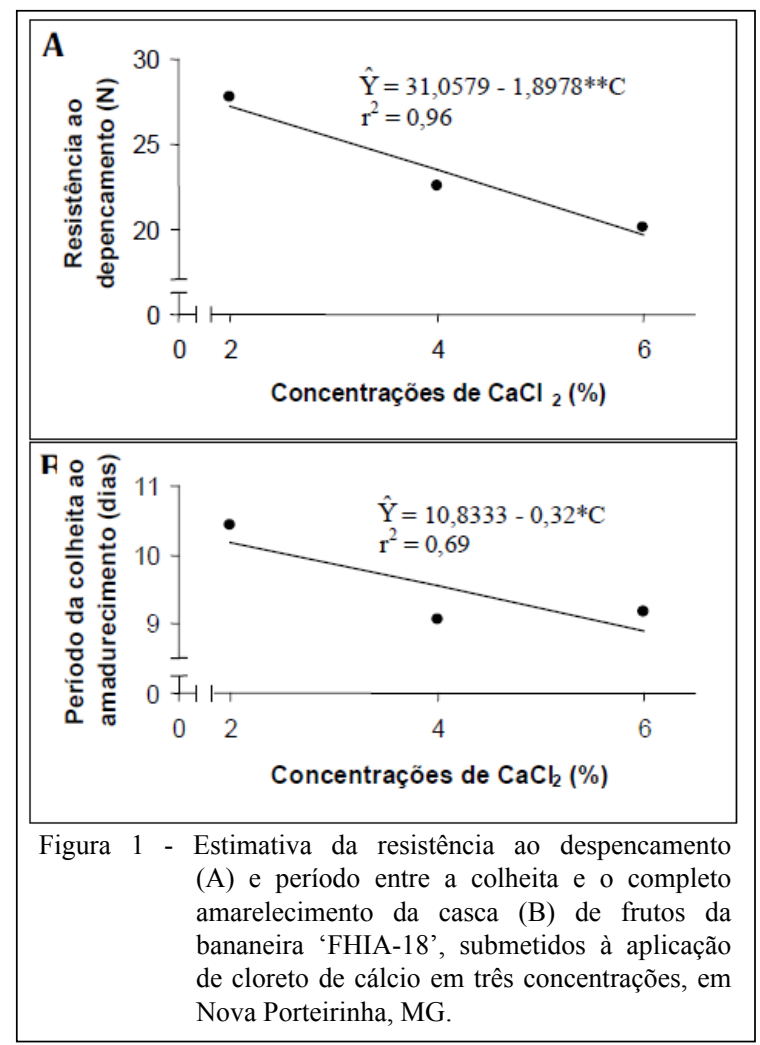


do fruto com o incremento das concentrações de cloreto de cálcio (Figura 1B), corroborando o resultado observado por SILVA (1999) no processo de amadurecimento da banana 'Pioneira'.

A aplicação das soluções de cloreto de cálcio nas doses de 2,4 e $6 \%$ não foi suficiente para afetar as características firmeza do fruto, porcentagem de perda de água do fruto da colheita ao estádio totalmente amarelo, tempo de emissão do cacho até a colheita, porcentagem de matéria seca da casca, da polpa e do pedicelo, teores de cálcio e magnésio na casca e teores de cálcio, magnésio e potássio na polpa. Os frutos perderam 19,75\% de água da colheita ao estádio 6 de maturação, e os cachos foram colhidos aos 123 dias após sua emissão. As porcentagens de água da casca, polpa e pedicelo dos frutos no estádio 6 de maturação foram de 85,80 e $69 \%$, respectivamente.

Quanto às épocas de aplicações, observou-se um aumento na firmeza do fruto com o aumento do número de aplicações. Em três aplicações de cloreto de cálcio, os frutos apresentaram-se mais consistentes do que com uma e duas aplicações, não diferindo da testemunha. Resultados semelhantes foram obtidos por BRACKMANN et al. (2010), que obtiveram maior firmeza dos frutos de maçãs submetidas à aplicação de maior número de aplicações de $\mathrm{CaCl}_{2}$.
O cálcio tem sido utilizado em tratamentos pré e pós-colheita para conferir resistência aos tecidos dos frutos, uma vez que os íons $\mathrm{Ca}^{++}$associamse com substâncias pécticas da parede celular e lamela média, impedindo a ação de enzimas como pectinametilesterase e poligalacturonase (AGHDAM et al., 2012). De acordo com XISTO et al. (2004), a aplicação de cálcio na pós-colheita em goiabas 'Pedro Sato', emprego de cálcio proporcionou atividades mais baixas de pectinametilesterase e poligalacturonase, promovendo um amaciamento menos intenso e mantendo a firmeza desses frutos. Estudos têm demonstrado que os tratamentos com cálcio em pós-colheita desaceleram o processo de amolecimento de frutos de banana (IMSABAI et al., 2006), maçã (JOHNSTON et al., 2002) e manga (MOOTOO, 1991). De acordo com os autores, o cálcio atua fixando as ligações cruzadas entre as moléculas de pectina e, portanto, mantendo a integridade da parede celular.

Nas avaliações do teor de nutrientes, detectou-se que frutos de cachos submetidos a uma aplicação de cloreto de cálcio 4\% 120 dias após a emissão apresentaram pedicelos com teor de cálcio de $5,6 \mathrm{~g} \mathrm{~kg}^{-1}$ (Tabela 1), enquanto os pedicelos de frutos de cachos submetidos a três aplicações exibiram menor teor de cálcio $\left(4,0 \mathrm{~g} \mathrm{~kg}^{-1}\right)$. O maior número de aplicações do produto deveria possibilitar a maior

Tabela 1 - Teores de cálcio na casca (CCA), na polpa (POC) e no pedicelo (PEC), de magnésio na casca (CMG), na polpa (POM) e no pedicelo (PEM) e de potássio na casca (CAK), na polpa (POK) e pedicelo (PEK) de frutos da bananeira 'FHIA-18' submetidos à aplicação de cloreto de cálcio em três concentrações $(\mathrm{C})$ e três épocas (EP), em Nova Porteirinha, MG.

\begin{tabular}{|c|c|c|c|c|c|c|c|c|c|}
\hline \multirow{2}{*}{$\begin{array}{l}\text { Época } \\
\text { EP1 }\end{array}$} & $2 \%$ & $4 \%$ & $6 \%$ & $2 \%$ & $4 \%$ & $6 \%$ & $2 \%$ & $4 \%$ & $6 \%$ \\
\hline & $4,3 \mathrm{a}$ & $4,2 \mathrm{a}$ & $4,3 \mathrm{a}$ & $0,6 \mathrm{a}$ & $0,7 \mathrm{a}$ & $0,6 \mathrm{a}$ & $3,7 \mathrm{a}$ & $5,6 \mathrm{a}$ & $5,3 \mathrm{a}$ \\
\hline EP2 & $3,5 \mathrm{a}$ & $3,9 \mathrm{a}$ & $4,2 \mathrm{a}$ & $0,6 \mathrm{a}$ & $0,6 \mathrm{a}$ & $0,6 \mathrm{a}$ & $4,5 \mathrm{a}$ & $5,2 \mathrm{ab}$ & $5,4 \mathrm{a}$ \\
\hline \multirow[t]{2}{*}{ EP3 } & $4,1 \mathrm{a}$ & $4,8 \mathrm{a}$ & $4,2 \mathrm{a}$ & $0,7 \mathrm{a}$ & $0,7 \mathrm{a}$ & $0,6 \mathrm{a}$ & $4,9 \mathrm{a}$ & $4,0 \mathrm{~b}$ & $5,3 \mathrm{a}$ \\
\hline & ------ & $\mathrm{MG}(\mathrm{g} \mathrm{k}$ & 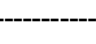 & ------- & $\mathrm{DM}(\mathrm{g} \mathrm{k}$ & -------- & ------ & EM ( $\mathrm{g} \mathrm{k}$ & ------ \\
\hline EP1 & $2,4 \mathrm{a}$ & $2,3 \mathrm{a}$ & $2,4 \mathrm{a}$ & $1,4 \mathrm{a}$ & $1,4 \mathrm{a}$ & $1,4 \mathrm{a}$ & $2,8 \mathrm{a}$ & $3,4 \mathrm{a}$ & $4,0 \mathrm{a}$ \\
\hline EP2 & $2,3 \mathrm{a}$ & $2,3 \mathrm{a}$ & $2,3 \mathrm{a}$ & $1,4 \mathrm{a}$ & $1,4 \mathrm{a}$ & $1,3 \mathrm{a}$ & $3,1 \mathrm{a}$ & $3,1 \mathrm{a}$ & $3,7 \mathrm{a}$ \\
\hline \multirow[t]{2}{*}{ EP3 } & $2,5 \mathrm{a}$ & $2,7 \mathrm{a}$ & $2,1 \mathrm{a}$ & $1,4 \mathrm{a}$ & $1,4 \mathrm{a}$ & $1,3 \mathrm{a}$ & $3,7 \mathrm{a}$ & $2,5 \mathrm{a}$ & $3,5 \mathrm{a}$ \\
\hline & & $\mathrm{AK}(\mathrm{g}$ l & י--י--י--ינ & --.-- & $\mathrm{OK}(\mathrm{g} \mathrm{kg}$ & --------. & -------- & $\mathrm{EK}(\mathrm{g} \mathrm{k}$ & \\
\hline EP1 & $57,1 \mathrm{a}$ & $54,3 \mathrm{a}$ & $78,5 \mathrm{a}$ & $19,2 \mathrm{a}$ & $19,2 \mathrm{a}$ & $17,3 \mathrm{a}$ & $34,1 \mathrm{a}$ & $36,2 \mathrm{a}$ & $38,2 \mathrm{a}$ \\
\hline EP2 & $46,2 \mathrm{a}$ & $61,0 \mathrm{a}$ & $67,0 \mathrm{ab}$ & $18,4 \mathrm{a}$ & $18,0 \mathrm{a}$ & $18,5 \mathrm{a}$ & $36,3 \mathrm{a}$ & $36,5 \mathrm{a}$ & $37,7 \mathrm{a}$ \\
\hline EP3 & $47,2 \mathrm{a}$ & $70,0 \mathrm{a}$ & $53,1 \mathrm{~b}$ & $18,4 \mathrm{a}$ & $18,3 \mathrm{a}$ & $18,2 \mathrm{a}$ & $33,1 \mathrm{a}$ & $33,4 \mathrm{a}$ & $39,2 \mathrm{a}$ \\
\hline
\end{tabular}

EP1 (120 dias após a emissão do cacho).

EP2 (90 e 120 dias após a emissão do cacho).

EP3 (60, 90 e 120 dias após a emissão do cacho).

As médias seguidas da mesma letra nas colunas e dentro da mesma característica não diferem entre si, a 5\% de probabilidade, pelo teste de Tukey. 
absorção do cálcio pelo fruto. No entanto, conforme descrito anteriormente, observou-se o contrário. $\mathrm{Na}$ concentração de $2 \%$ de $\mathrm{CaCl}_{2}$, apesar de demonstrar tendência de aumento do teor de cálcio no pedicelo com o incremento do número de aplicações, não se verificaram diferenças significativas. Aplicando $6 \%$ de cloreto de cálcio e variando o número de aplicações, o teor de cálcio no pedicelo praticamente manteve-se constante (Tabela 1).

Os teores de cálcio da casca e da polpa dos frutos não foram alterados significativamente com as concentrações e épocas de aplicação do produto. Os valores médios de cada tratamento para tais características foram maiores do que os das testemunhas, entretanto sem diferenças significativas. Isso demonstra que o cálcio não foi absorvido efetivamente na casca e polpa do fruto, em decorrência da aspersão do cloreto de cálcio. Em tratamento pós-colheita de maçãs, o cálcio é absorvido pelo fruto, principalmente através das lenticelas, ressaltando-se que espaços ou aberturas na cutícula e epiderme também devem ser importantes portas de entrada, especialmente quando o fruto se encontra mais maduro (GLENN \& POOVAIAH, 1985; CONWAY et al., 1992). No entanto, apenas a imersão das maçãs em solução contendo cálcio não tem sido suficiente para mover o cálcio para dentro do fruto. Para a efetiva penetração do cálcio no fruto, torna-se necessário submetê-lo a determinada pressão de infiltração (CONWAY et al., 1992). Talvez para banana haja necessidade de tratamentos semelhantes. POOVAIAH (1986) demonstrou o uso de uma unidade móvel para tratamentos de frutos com cálcio por infiltração sob pressão. Segundo ele, a quantidade de cálcio absorvido é controlada pela concentração deste na solução, pelo grau de vácuo ou pressão aplicada, pelo tempo de submersão do produto na solução e pela temperatura da solução.

É importante ressaltar que a água utilizada na irrigação das bananeiras apresentou teor de $200 \mathrm{mg}$ $\mathrm{L}^{-1}$ de $\mathrm{CaCO}_{3}$, valor esse elevado em relação ao da água fluvial normalmente utilizada para irrigação dos demais plantios localizados no distrito de irrigação do rio Gorutuba, que apresenta $32 \mathrm{mg} \mathrm{L}^{-1}$ de $\mathrm{CaCO}_{3}$. Esse fato indica que o solo já apresentava boa disponibilidade de cálcio para absorção pelas raízes das plantas. Consequentemente, não se detectaram diferenças significativas nos teores de cálcio na casca e polpa de frutos submetidos a aplicações na précolheita de $\mathrm{CaCl}_{2}$.

Os teores médios de cálcio na casca $\left(3,40 \mathrm{~g} \mathrm{~kg}^{-1}\right)$ e na polpa $\left(0,53 \mathrm{~g} \mathrm{~kg}^{-1}\right)$ apresentados pela testemunha foram muito superiores aos teores médios de cálcio extraídos pelos frutos de bananeira $(0,21 \mathrm{~g}$ $\mathrm{kg}^{-1}$ ) relatados por ALVES (1999), em trabalho com diferentes variedades, e aos encontrados por LÓPEZ \& ESPINOSA (1995) e SILVA (1999), cujas médias foram, respectivamente, de $0,16 \mathrm{~g} \mathrm{~kg}^{-1}$ e $0,15 \mathrm{~g} \mathrm{~kg}^{-1}$.

Os teores de magnésio na casca, polpa e pedicelo e de potássio na polpa e pedicelo não se alteraram significativamente, em função das três épocas de aplicação de $\mathrm{CaCl}_{2}$ (Tabela 1). Observou-se que uma aplicação de cloreto de cálcio aos 120 dias após a emissão do cacho, na concentração de $6 \%$, proporcionou maior teor de potássio na casca, com $78,5 \mathrm{~g} \mathrm{~kg}^{-1}$, enquanto, com três aplicações, obteve-se o menor teor, com 53,1 $\mathrm{g} \mathrm{kg}^{-1}$ (Tabela 1). Isso pode ser explicado pelo antagonismo entre o $\mathrm{K}$ e o $\mathrm{Ca}$.

A interação entre nutrientes em cultivos de bananeira tem sido bastante estudada, principalmente entre $\mathrm{K}, \mathrm{Ca}$ e $\mathrm{Mg}$, podendo ser positiva e chamada de sinergismo ou negativa e considerada como antagonismo. Sintomas de deficiência de K são observados quando $\mathrm{Ca}$ e $\mathrm{Mg}$ apresentam valores elevados (SILVA1999; LÓPEZ \& ESPINOSA, 1995). De acordo com PUTRA et al. (2010), o alongamento das células e o enfraquecimento da parede celular após o amadurecimento pode ser manejado por meio da aplicação de alguns nutrientes, como: $\mathrm{Ca}, \mathrm{Mg}, \mathrm{B}$ e Si durante o período de cultivo. Segundo o autor, estes elementos são essenciais na manutenção da integridade das células e, em caso de deficiência, podem incrementar o despencamento dos frutos.

Por meio do ajuste de equações de regressão, observaram-se os efeitos das concentrações de cálcio em cada época de aplicação, desdobrando apenas as interações significativas. Realizando apenas uma aplicação de $\mathrm{CaCl}_{2}$ aos 120 dias após a emissão do cacho, observou-se incremento linear dos teores de cálcio no pedicelo com o aumento das concentrações (2, 4 e 6\%), enquanto, com três aplicações, detectouse comportamento quadrático e, com duas aplicações, não houve efeito. Entretanto, o incremento dos teores de cálcio conferiu menor resistência ao despencamento (Figura 1A), não justificando a aplicação na pré-colheita desse nutriente em cachos de bananeira.

Aplicando o $\mathrm{CaCl}_{2}$ uma vez aos 120 dias e duas vezes aos 90 e 120 dias, observou-se comportamento linear positivo, enquanto que, com a aplicação de três vezes, o comportamento foi quadrático para teores de K na casca. Nesses casos, verificou-se antagonismo da aplicação de $\mathrm{Ca}$ em relação ao $\mathrm{K}$ apenas com três aplicações e valores acima de $4 \%$ de $\mathrm{CaCl}_{2}$. Realizando três aplicações de $\mathrm{CaCl}_{2}$, observou-se que os teores de magnésio no 
pedicelo resultaram em comportamento quadrático, com redução de até $4 \%$ e aumento de até $6 \%$. Com duas aplicações, não houve efeito, enquanto que com uma aplicação ocorreu incremento linear positivo.

\section{CONCLUSÃO}

Os frutos apresentaram maior firmeza com três aplicações de $\mathrm{CaCl}_{2}$. Os teores de cálcio da casca e da polpa não foram alterados significativamente com as concentrações e épocas de aplicações do $\mathrm{CaCl}_{2}$, indicando que o cálcio não é absorvido efetivamente pelo fruto. A aplicação pré-colheita de cloreto de cálcio em diferentes concentrações e épocas não reduz o despencamento dos frutos da bananeira 'FHIA-18'.

\section{AGRADECIMENTOS}

À Fundação de Amparo a Pesquisa do Estado de Minas Gerais (FAPEMIG), pela concessão de bolsas de estudo.

\section{REFERÊNCIAS}

AGHDAM, M.S. et al. The language of calcium in postharvest life of fruits, vegetables and flowers. Scientia Horticulturae, v.144, p.102-115, 2012. Disponível em: <http://dx.doi.org/10.1016/j. scienta.2012.07.007>. Acesso em: 24 jan. 2013

AGRAVANTE, J.U. et al. Packging and transport of 'Cuarenta Dias' banana. Philippine Agricultural Science, v.83, n.3, p.244-245, 2000.

ALVES, E.J. A cultura da banana: aspectos técnicos, socioeconômicos e agroindustriais. 2.ed. Brasília: Embrapa-SPI/ Cruz das Almas; Embrapa-CNPMF, 1999. 585p.

BRACKMANN, A. et al. Aplicações pré-colheita de cálcio na qualidade pós-colheita de maçãs 'Fuji'. Ciência Rural, v.40, n.6, p.1435-1438, 2010. Disponível em: <http://www.scielo.br/ scielo.php?pid $=$ S0103-84782010000600032\&script $=$ sci_arttext $>$. Acesso em: 23 jan. 2013. doi:10.1590/S0103-84782010000600032.

CONWAY, W.S. et al. Calcium treatment of apples and potatoes to reduce postharvest decay. Plant Disease, v.76, n.4, p. 329-334, 1992.

CORDEIRO, Z.M.; MOREIRA, R.S. A bananicultura brasileira. In: REUNIÃO INTERNACIONAL DA ACORBAT, 17., 2006, Joinville, SC. Anais... Joinville: ACORBAT/ACAFRUTA, 2006, v.1, p.36-47.

DONATO, S.L.R. et al. Comportamento fitotécnico da bananeira 'Prata-Anã' e de seus híbridos. Pesquisa Agropecuária Brasileira, v.44, n.12, p.1608-1615, 2009. Disponível em: <http:// dx.doi. org/10.1590/S0100-204X2010000900007>. Acesso em: 25 dez. 2012. doi:10.1590/S0100-204X2010000900007.

ESGUERRA, E.B. et al. Responses of 'Señorita' and 'Cavendish' bananas to postharvest ethanol treatment. ASEAN Food Journal, v.8, n.2, p.6-9, 1993.

GLENN, G.M.; POOVAIAH, B.W. Cuticular permeability to calcium compounds in 'Golden Delicious' apple fruit. Journal of American Society for Horticultural Science, v.110, n.2, p.192, 1985. Disponível em: <http://dx.doi.org/10.1016/030442389090052-G>. Acesso em: 20 ago. 2013. doi:10.1016/030442389090052

HOLDERNESS, M. et al. Organic banana 2000: towards an organic banana initiative in the Caribbean. Montpellier: INIBAP, 2000. 174p. (Report of the International workshop on the production and marketing of organic bananas by smallholder farmers).

IMSABAI, W. et al. Physiological and biochemical changes during banana ripening and finger drop. Postharvest Biological Technology, v.38, p.211-216, 2006. Disponível em: <http://dx.doi. org/10.1016/j.postharvbio.2005.10.001>. Acesso em: 15 out. 2013. doi:10.1016/j.postharvbio.2005.10.001.

JESUS, O.N. et al. Characterization of recommended banana cultivars using morphological and molecular descriptors. Crop Breeding and Applied Biotechnology, v.9, p.164-173, 2009

JOHNSTON, J.W. et al. Postharvest softening of apple (Malus domestica) fruit: a review. New Zealand Journal Crop Horticultural Science, v.30, p.145-160, 2002. Disponível em: $<$ http://dx.doi.org/10.1080/01140671.2002.9514210>. Acesso em: 16 out. 2013. doi:10.1080/01140671.2002.9514210.

LÓPEZ, A.; ESPINOSA, J. Manual de nutricion y fertilización del banano. Quito: INPOFOS, 1995. 82p.

MOOTOO, A. Effect of postharvest calcium chloride dips on ripening changes in 'Julie' mangoes. Tropical Science, v.31, p.243-248, 1991.

PAULL, R.E. Ethylene, storage and ripening temperatures affect 'Dwarf Brazilian' banana finger drop. Postharvest Biology Biotechnology, v.8, p.65-74, 1996. Disponível em: <http://dx.doi. org/10.1016/0925-52149500058-5>. Acesso em: 10 out. 2013. doi:10.1016/0925-52149500058-5.

PEREIRA, M.C.T. et al. Suscetibilidade à queda natural e caracterização dos frutos de diversos genótipos de bananeiras. Revista Brasileira de Fruticultura, v.26, n.3, p.499-502, 2004. Disponível em: <http://dx.doi.org/10.1590/S010029452004000300030>. Acesso em: 10 jan 2012. doi:10.1590/ S0100-29452004000300030.

POOVAIAH, B.W. Role of calcium in prolonging storage life of fruits and vegetables. Food Technology, v.40, p.86-89, 1986.

PUTRA, E.T.S. et al. Weak neck of Musa sp. cv. 'Rastali': a review on its genetics, crop nutrition, and postharvest. Journal of Agronomy, v.9, n.2, p.45-51, 2010. Disponível em: <http:// dx.doi.org/10.3923/ja.2010.45.51>. Acesso em: 10 jan. 2012. doi:10.3923/ja.2010.45.51

RAO, D.V.R.; CHUNDAWAT, B.S. Chemical regulation of ripening in banana bunches cv. 'Lacatan' at non-refrigerated temperatures. Haryana Journal Horticultural Science, v.20, p.6-11, 1991. Disponível em: <http://dx.doi.org/10.1111/1541-4337.12025>. Acesso em: 10 jan. 2012. doi:10.1111/1541-4337.12025.

SAENGPOOK, C. et al. Effects of relative humidity on banana fruit drop. Postharvest Biology and Technology, v.45, n.1, p.151-154, 2007. Disponível em: <http://dx.doi.org/10.1016/j. postharvbio.2007.02.004>. Acesso em: 10 jan. 2013. doi:10.1016/j. postharvbio.2007.02.004. 
SALAZAR, B.M.; SERRANO, E.P. Etiology and postharvest control of finger drop disorder in 'Cuarenta Dias' banana (Musa acuminata AA Group). Philippine Agricultural Science, v.96, n.2, p.163-171, 2013.

SANTOS, B.H.C. et al. Controle de Meloidogyne javanica em mudas de bananeira 'Prata-anã' por compostos orgânicos. Revista Brasileira de Fruticultura, v.35, n.2, p.650-656, 2013. Disponível em: <http://dx.doi.org/10.1590/S010029452013000200038>. Acesso em: 20 set. 2013. doi:10.1590/ S0100-29452013000200038.

SANTOS, S.C. et al. Caracterização morfológica e avaliação de cultivares de bananeira resistentes a Sigatoka Negra (Mycosphaerella fijiensis Morelet) no sudoeste goiano. Revista
Brasileira de Fruticultura, v.28, n.3, p.449-453, 2006. Disponível em: <http://dx.doi.org/10.1590/S0100-29452006000300024>. Acesso em: 10 fev. 2013. doi:10.1590/S0100-29452006000300024.

SILVA, C. L. Aplicação pós-colheita de cálcio em frutos de bananeira (Musa sp.) cultivar 'Pioneira'. 1999. 56f. Dissertação (Mestrado em Ciências Agrárias) - Curso de Pós-graduação em Agronomia, Universidade Federal da Bahia.

XISTO, A.L.R.P. et al. Textura de goiabas 'Pedro Sato' submetidas à aplicação de cloreto de cálcio. Ciência e Agrotecnologia, v.28, n.1, p.113-118, 2004. Disponível em: <http://dx.doi.org/10.1590/ S1413-70542004000100015>. Acesso em: 10 set. 2013. doi:10.1590/S1413-70542004000100015. 